\title{
Fermi Level Stabilization Energy in Cadmium Oxide
}

\author{
D. T. Speaks ${ }^{1,2}$, M. A. Mayer ${ }^{1,2}$, K. M. Yu ${ }^{1}$, S. S. Mao ${ }^{3,4}$, E. E. Haller ${ }^{1,2}$ and W. \\ Walukiewicz ${ }^{1}$ \\ ${ }^{1}$ Materials Sciences Division, Lawrence Berkeley National Laboratory, Berkeley, \\ California 94720, USA \\ ${ }^{2}$ Department of Materials Science \& Engineering, University of California at Berkeley, \\ Berkeley California 94704, USA \\ ${ }^{3}$ Environmental Energy Technologies Division, Lawrence Berkeley National Laboratory, \\ Berkeley, California 94720, USA \\ ${ }^{4}$ Department of Mechanical Engineering, University of California at Berkeley, Berkeley, \\ California 94704, USA
}

\section{ABSTRACT}

We have studied the effects of high concentrations of native point defects on the electrical and optical properties of $\mathrm{CdO}$. The defects were introduced by irradiation with high energy $\mathrm{He}^{+}, \mathrm{Ne}_{+}, \mathrm{Ar}^{+}$and $\mathrm{C}^{+}$ions. Increasing the irradiation damage with particles heavier than $\mathrm{He}^{+}$increases the electron concentration until a saturation level of $5 \times 10^{20}$ $\mathrm{cm}^{-3}$ is reached. In contrast, due to the ionic character and hence strong dynamic annealing of $\mathrm{CdO}$, irradiation with much lighter $\mathrm{He}^{+}$stabilizes the electron concentration at a much lower level of $1.7 \times 10^{20} \mathrm{~cm}^{-3}$. A large shift of the optical absorption edge with increasing electron concentration in irradiated samples is explained by the Burstein-Moss shift corrected for electron-electron and electron-ion interactions. The saturation of the electron concentration and the optical absorption edge energy are consistent with a defect induced stabilization of the Fermi energy at $1 \mathrm{eV}$ above the conduction band edge. The result is in a good agreement with previously determined Fermi level pinning energies on $\mathrm{CdO}$ surfaces. The results indicate that $\mathrm{CdO}$ shares many similarities with $\mathrm{InN}$, as both materials exhibit extremely large electron affinities and an unprecedented propensity for n-type conductivity. 


\section{INTRODUCTION}

There has been increasing interest in group II-oxides, a class of semiconductors analogous to the very extensively studied and commercially successful group-III nitrides. $\mathrm{ZnO}$ has been thoroughly investigated but less attention has been paid to other group-II oxides. Although $\mathrm{CdO}$ has been synthesized by different methods, its properties are not very well established ${ }^{123456}$. There is still controversy regarding the nature, direct or indirect, of the fundamental band gap of this material. Recent angle-resolved photoemission spectroscopy (ARPES) studies have shown that the Fermi energy on the free $\mathrm{CdO}$ surface is pinned at about $1.15 \mathrm{eV}$ above the conduction band edge $\left(\mathrm{E}_{\mathrm{C}}\right)^{7}$. This resembles the extensively studied case of InN where the Fermi energy was found to be pinned deeply in the conduction band at $\mathrm{E}_{\mathrm{C}}+0.9 \mathrm{eV}$. It is important to note that, as in InN, the surface Fermi level pinning energy for semiconductors is expected to be the same as the bulk Fermi level stabilization energy in heavily damaged materials ${ }^{89}$. This important result is a consequence of the amphoteric defect model, originally developed for GaAs in the late 1980's, which has since been successfully applied to many other semiconductors $^{8} 91011$. It was shown that the nature, donor or acceptor, of the native defects in semiconductors is determined by the position of the Fermi energy $\left(\mathrm{E}_{\mathrm{F}}\right)$ with respect to the Fermi level stabilization energy $\left(\mathrm{E}_{\mathrm{FS}}\right)$. The dominant native defects in a semiconductor are donors (acceptors) if $\mathrm{E}_{\mathrm{F}}<\mathrm{E}_{\mathrm{FS}}\left(\mathrm{E}_{\mathrm{F}}>\mathrm{E}_{\mathrm{FS}}\right)$. $\mathrm{E}_{\mathrm{F}}$ stabilizes when the formation rates of both donors and acceptors are equal, and this occurs when $\mathrm{E}_{\mathrm{F}}=\mathrm{E}_{\mathrm{FS}}$. It should be noted that it has been shown in the case of $\mathrm{GaAs}$ for $\mathrm{E}_{\mathrm{F}}<\mathrm{E}_{\mathrm{FS}}\left(\mathrm{E}_{\mathrm{F}}>\mathrm{E}_{\mathrm{FS}}\right)$ both cation and anion site vacancies are donors (acceptors). Particle irradiation has been used

previously in InN and other materials to effectively control the electronic properties ${ }^{12}$ 
because ions produce a uniform distribution of point defects wherethe defects tend to be acceptors or donors depending on the position of $\mathrm{E}_{\mathrm{F}}$ relative to $\mathrm{E}_{\mathrm{FS}}$.

In a striking contrast to the surface pinning at $\mathrm{E}_{\mathrm{F}}=1 \mathrm{eV}$ in $\mathrm{CdO}$ determined by $\mathrm{ARPES}^{7}$, a recent study has shown that an intentional introduction of native point defects using irradiation with $1 \mathrm{MeV} \mathrm{He}^{+}$ions led to stabilization of the bulk Fermi energy at about $\mathrm{E}_{\mathrm{C}}+0.4 \mathrm{eV}$ in $\mathrm{CdO}^{13}$ which is almost 3 times lower than the surface Fermi level pinning energy. To address this inconsistency in the bulk and surface pinning of the Fermi level and to better understand the properties of native defects in $\mathrm{CdO}$, we have performed systematic studies of electrical and optical properties of $\mathrm{CdO}$ irradiated with high energy ions of different mass and energy. We show that the irradiation-induced pinning energy depends on the damage introduction rate. A good agreement between surface Fermi level pinning and bulk Fermi level stabilization energy is achieved for high introduction rate of damage using heavy ions such as $\mathrm{Ne}$ and Ar that eliminates damage self-healing effects.

\section{EXPERIMENTAL DETAILS}

CdO films were deposited by pulsed laser deposition (PLD) on c-plane sapphire substrates. A Lambda Physik KrF excimer pulsed laser operating at $248 \mathrm{~nm}$ with a pulse width of 10 nanoseconds, energy fluence of $160 \mathrm{~mJ}$ and repetition rate of $5 \mathrm{~Hz}$ was used. Sapphire substrates, $6 \mathrm{~mm}$ by $6 \mathrm{~mm}$, were first cleaned with acetone followed by methanol. After mounting in the chamber, the substrates were baked at $500^{\circ} \mathrm{C}$ for 30 minutes to remove any residual contamination. A distance of $6 \mathrm{~cm}$ was maintained between the substrate and the pure $\mathrm{Cd}$ target during deposition. The films were deposited at $270^{\circ} \mathrm{C}$ with 40,000 laser pulses. Prior to deposition, the chamber was pumped down to 
$1 \times 10^{-6}$ torr. During deposition the background pressure was maintained at a constant $4.2 \times 10^{-4}$ torr by flowing $\mathrm{O}_{2}$.

The crystalline structure of the films was determined by X-ray diffraction (XRD). Film stoichiometry and thickness were measured by Rutherford backscattering (RBS) using a $2 \mathrm{MeV} \mathrm{He}{ }^{+}$ion beam. The optical absorption edges were measured using a Perkin-Elmer Lambda 950 Spectrophotometer. Electron concentrations and mobilities were determined by Hall Effect in the Van der Pauw configuration using an Ecopia HMS-3000 system with a 0.6 Tesla magnetic field.

In order to compare the degree of irradiation damage among several different ions, the displacement damage dose methodology developed by the Naval Research Laboratory was used ${ }^{14}{ }^{15}$. The displacement damage dose $\left(D_{d}\right.$ in units of $\left.\mathrm{MeV} / \mathrm{g}\right)$ is defined as the non-ionizing energy loss (NIEL) multiplied by the dose. The NIEL for different ions in $\mathrm{CdO}$ were modeled using the SRIM (the Stopping and Range of Ions in Matter) program ${ }^{16}$. Point defects in $\mathrm{CdO}$ films were generated by irradiating the sample with $120 \mathrm{keV} \mathrm{Ne}^{+}, 33 \mathrm{keV} \mathrm{C}^{+}, 80 \mathrm{keV} \mathrm{Ar}^{+}$and $2 \mathrm{MeV} \mathrm{He}^{+}$particles with displacement damage doses ranging from $9.3 \times 10^{14}$ to $1.0 \times 10^{17} \mathrm{MeV} / \mathrm{g}$.

\section{RESULTS}

Figure 1 shows the XRD spectrum of a $\mathrm{CdO}$ film deposited on sapphire. Only the $\mathrm{CdO}(002)$ peak $\left(2 \theta=38.32^{\circ}\right)$ is observed together with the sapphire (0006) substrate peak $\left(2 \theta=41.70^{\circ}\right)$. This suggests that the $\mathrm{CdO}$ film grows in the 001 preferred direction. The preferred growth direction is also confirmed by channeling RBS measurements that show a channeling yield $\chi \sim 0.5$. This preferred growth direction is expected because it is the 
highest density plane and thus should have the lowest surface energy ${ }^{1}$. The narrow (002) diffraction peak from the $\mathrm{CdO}$ also indicates a large grain size. Using the Scherrer equation, the grain size is found to be on the order of $150 \mathrm{~nm}$. This is on the same order of film thickness as measured by RBS, i.e. $130 \mathrm{~nm}$.

Hall Effect measurements show that the as-grown film is n-type with an electron concentration of $9 \times 10^{19} \mathrm{~cm}^{-3}$ and mobility of $89 \mathrm{~cm}^{2} / \mathrm{V}$-s. Due to the large electron affinity of the material, $\mathrm{CdO}$ films have a clear tendency to be n-type. In fact, all reported $\mathrm{CdO}$ films are n-type with electron concentration on the order of $10^{19}-10^{20} / \mathrm{cm}^{3}$. The mobility of $89 \mathrm{~cm}^{2} / \mathrm{V}$-s measured in our film is higher than most CdO thin films reported in the literature with comparable electron concentration. The XRD, RBS and electrical measurements indicate that the quality of these as-grown $\mathrm{CdO}$ films is rather high.

The XRD spectrum of the $\mathrm{CdO}$ irradiated by $120 \mathrm{keV} \mathrm{Ne}^{+}$ions to a total fluence of $1.2 \times 10^{14} \mathrm{~cm}^{-2}$ (corresponding to a $D_{\mathrm{d}} \sim 6.7 \times 10^{16} \mathrm{MeV} / \mathrm{g}$ ) is also shown in Figure 1. We note that this irradiation condition gives rise to saturation of electrical properties in $\mathrm{CdO}$. First we observe that the high irradiation dose does not amorphize the film as the (002) diffraction peak is still present, though slightly shifted to a lower value. This suggests that the point defects created by $\mathrm{Ne}$ irradiation result in an increase of the lattice constant, a behavior also observed in $\mathrm{InN}^{9}$.

The effect of irradiation on the electron concentration is shown in Figure 2. For comparison, irradiation effects in $\mathrm{InN}$ and the recent reported results by King et al. on $\mathrm{CdO}$ are also shown ${ }^{13}$. Similar to the case of $\mathrm{InN}$, irradiation increases the free carrier concentrations in all $\mathrm{CdO}$ films. Regardless of the ion used, saturation of the electron 
concentration is observed for $\mathrm{CdO}$ as the displacement damage dose increases to $>2 \times 10^{16}$ $\mathrm{MeV} / \mathrm{g}$. These results are consistent with the amphoteric defect model (ADM) ${ }^{8}$ assuming that the Fermi level stabilization energy, $\mathrm{E}_{\mathrm{FS}}$ in $\mathrm{CdO}$ lies well above the conduction band minimum. Therefore, the irradiation-induced point defects are predominantly donors leading to an increase of the electron concentration and an upward shift of the Fermi energy, $\mathrm{E}_{\mathrm{F}}$ towards $\mathrm{E}_{\mathrm{FS}}$. For $\mathrm{E}_{\mathrm{F}}=\mathrm{E}_{\mathrm{FS}}$, introduction of new defects does not change the electron concentration or the Fermi energy as the formation rates of donor- and acceptorlike defects are equal.

Results in Figure 2 show that the irradiation with $\mathrm{C}^{+}, \mathrm{Ne}+$ and $\mathrm{Ar}^{+}$ions leads to an increase in electron concentration and the eventual saturation of the electron concentration at $N_{\text {sat }} \sim 4.5-5.0 \times 10^{20} \mathrm{~cm}^{-3}$ with the damage doses $\mathrm{D}_{\mathrm{d}}>2 \times 10^{16} \mathrm{MeV} / \mathrm{g}$. Electron concentration is related to the Fermi level according to

$$
n=\frac{1}{3 \pi^{2}} \int_{E_{C} / k_{B} T}^{\infty} \frac{\exp \left(z-E_{F} / k_{B} T\right)}{1+\exp \left(z-E_{F} / k_{B} T\right)^{2}} k^{3}(z) d z
$$

where $\mathrm{k}_{\mathrm{B}}$ is the Boltzmann constant, $\mathrm{z}=\mathrm{E}_{\mathrm{c}} / \mathrm{k}_{\mathrm{B}} \mathrm{T}, \mathrm{E}_{\mathrm{F}}$ is the electron energy and $\mathrm{E}_{\mathrm{C}}$ is the energy of the conduction band edge. The nonparabolic dispersion relation for $\mathrm{CdO}$ was derived from Kane's two band $\mathbf{k}^{*} \mathbf{p}$ model $^{17}$ :

$$
E_{c}(k)=\frac{\hbar^{2} k^{2}}{2 m_{0}}-\frac{E_{g}}{2}+\sqrt{\left(\frac{E_{g}}{2}\right)^{2}+\left(\frac{E_{g} \hbar^{2} k^{2}}{2 m_{0}^{*}}\right)}
$$

The carrier concentration as a function of Fermi energy, $\mathrm{E}_{\mathrm{F}}$, is calculated using Eq. 1 with $\mathrm{k}$ dependence on $\mathrm{E}$ obtained by solving Eq 2. Assuming the conduction band edge electron effective mass of $\mathrm{m}_{0} *=0.21 \mathrm{~m}_{0}$ we find that the saturation electron concentration $\mathrm{N}_{\mathrm{sat}} \sim 5 \times 10^{20} \mathrm{~cm}^{-3}$ corresponds to $\mathrm{E}_{\mathrm{F}}$, and thus also $\mathrm{E}_{\mathrm{FS}}$, located deeply in the conduction 
band at $\sim \mathrm{E}_{\mathrm{C}}+1 \mathrm{eV}$. This location of $\mathrm{E}_{\mathrm{FS}}$ is in a good agreement with the recently reported surface level pinning energy measured by photoelectron spectroscopy ${ }^{7}$. These results confirm the basic assertion of the ADM that the same mechanism is responsible for the Fermi level pinning on the surface and the Fermi level stabilization in the bulk of CdO. Our heavy particle irradiation experiments point to certain similarity of the defect behavior in $\mathrm{CdO}$ and $\mathrm{InN}^{9}$. In both instances damage produces high concentration n-type material with $\mathrm{E}_{\mathrm{FS}}$ located almost $1 \mathrm{eV}$ above the conduction band edge. This is in a stark contrast with most semiconductors where $\mathrm{E}_{\mathrm{FS}}$ is located in the band gap and irradiation produces high resistivity material.

Interestingly, the results in Figure 2 show a large difference in the dependence of the saturation electron concentration on the damage produced by a heavy ion and $\mathrm{He}^{+}$ irradiation. In our $\mathrm{He}^{+}$irradiated sample the electron concentration saturates at about $1.7 \times 10^{20} \mathrm{~cm}^{-3}$. This is very close to the electron concentration saturation of $2 \times 10^{20} \mathrm{~cm}^{-3}$ reported by King et al. ${ }^{13}$ for $\mathrm{CdO}$ irradiated with $1 \mathrm{MeV} \mathrm{He}^{+}$ions but much lower than the $N_{\text {sat }}$ obtained with the heavy ion irradiation. In contrast to $\mathrm{CdO}$, irradiation of $\mathrm{InN}$ with $2 \mathrm{MeV} \mathrm{He}^{+}$, as well as with $\mathrm{Ne}^{+}$, stabilizes the Fermi level at $\mathrm{E}_{\mathrm{C}}+0.9 \mathrm{eV}$, the same as Fermi level pinning position on InN surfaces. A likely cause of this discrepancy in the value of $N_{\text {sat }}$ obtained from $\mathrm{He}$ and heavy ion irradiation in $\mathrm{CdO}$ is the large difference in the damage production rate for these ions. We note that the NIEL of a $120 \mathrm{keV} \mathrm{Ne}^{+}$ion in $\mathrm{CdO}$ is over 500 times higher than that of a $2 \mathrm{MeV} \mathrm{He}^{+}$ion. This means that for similar irradiation currents, the defect production rate in $\mathrm{CdO}$ for a heavy ion $\left(\mathrm{Ne}^{+}, \mathrm{C}+\right.$, $\left.\mathrm{Ar}^{+}\right)$is at least 2 orders of magnitude higher than for much lighter He ions. Therefore, irradiation with heavy ions produces a high density of vacancies and interstitials in the 
ion path. Fast diffusing interstitials form stable clusters that act as sinks for newly formed interstitials leaving the vacancies which, as has been discussed in the introduction, are expected to be electrically active donors for $\mathrm{E}_{\mathrm{F}}<\mathrm{E}_{\mathrm{FS}}$ and stabilize the Fermi energy at $\mathrm{E}_{\mathrm{FS}}$. In the case of $\mathrm{He}^{+}$irradiation, the lower density of defects in the path of the high energy ion prevents precipitation of interstitials that remain isolated in the crystal lattice. Subsequently produced vacancies have a large chance to be annihilated by these interstitials. At high enough irradiation doses, a balance between production and annihilation of the vacancies is established resulting in saturation of the electron concentration and stabilization of the Fermi energy below $\mathrm{E}_{\mathrm{FS}}$. Dynamic annealing becomes much more significant for more ionic materials such as $\mathrm{CdO}$. This empirical relationship between bond ionicity and degree of dynamic annealing has been known since the 1970s', however the exact physical mechanisms that cause this phenomena are not well established ${ }^{18} 19$. However, Kucheyev states that the two most likely mechanisms are an increase in electrostatic energy associated with substitutional disorder and electrostatically lowering energy barriers for defect interaction processes as ionicity increases ${ }^{20}$. What is known is that very ionic materials, such as $\mathrm{ZnO}$, exhibit strong dynamic annealing, and $\mathrm{ZnO}$ and $\mathrm{CdO}$ have comparable ionicity ${ }^{21}$. In addition, similar dynamic annealing behavior has been recently observed in Zr-irradiated pure and yttria-stabilized zirconia ${ }^{22}$ where dynamic annealing was found to be especially significant in the presence of oxygen vacancies, a condition that is also present in CdO.

In order to further evaluate effects of irradiation on the properties of $\mathrm{CdO}$, we have measured optical properties and analyzed the electron concentration-dependent position of the optical absorption edge in terms of the Burstein-Moss shift ${ }^{23}$. Figure 3 
shows the optical absorption curves for the same $\mathrm{CdO}$ thin film which was sequentially irradiated with increasing $\mathrm{Ne}^{+}$ion dose.

A clear blue shift in the absorption edge is observed with increasing irradiation dose. Notice that the initially small increases in irradiation dose result in large shifts in the position of the absorption edge. However, as the dose approaches the saturation level the shift in the absorption curves becomes smaller until almost no movement in the absorption edge is observed even for doubling the irradiation dose. The absorption edge shift to higher energy is well described by the Burstein-Moss shift: electrons filling the bottom of the conduction band result in a blue shift in the optical absorption edge energy due to the Fermi exclusion principle. This is depicted qualitatively in Figure 4a: the red line (labeled $\mathrm{E}_{\mathrm{F}}$ ) represents the Fermi level above the conduction band minimum. As the Fermi level moves higher in the conduction band with the addition of electrons, it eventually stabilizes at $\mathrm{E}_{\mathrm{FS}}$ represented by the solid green line in Figure 4a.

To quantify this shift, we compare the experimental data with calculated absorption edges using the $\mathrm{CdO}$ band structure in conjunction with $\mathrm{E}_{\mathrm{F}}$ values directly deduced from Hall effect electron concentrations. Because of the large electron concentrations, the position of the conduction band edge and also the Burstein-Moss shift are expected to be affected by electron-electron and electron-ion interactions. First, the experimental edge was extracted for each data curve shown in Figure 3 by fitting with an analytical expression because a standard $\alpha^{2}$ linear approximation does not necessarily apply in a complex case ${ }^{24}$. A standard absorption expression for optical absorption was convoluted with a Gaussian curve to account for experimental broadening of the absorption edge: 


$$
\alpha\left(E^{\prime}\right)=\int_{-\infty}^{\infty} A\left(E-E_{A E}\right)^{1 / 2} \frac{\operatorname{Exp}-\left(\frac{\left(E^{\prime}-E\right)}{\Delta}\right)^{2}}{\Delta \sqrt{\pi}} d E
$$

where $\mathrm{A}$ is a scaling factor and $\Delta$ is the broadening parameter. For each curve, the value of the energy absorption edge, $\mathrm{E}_{\mathrm{AE}}$ labeled as "Optical Absorption at $\mathrm{k}_{\mathrm{F}}$ " in Figure 4a was determined from fitting Eq. 3 to the experimental data shown in Figure 3. With values of $\Delta$ of $0.3 \mathrm{eV}$ or less, we were able to obtain good fits to the first 6 absorption curves and extract the absorption edges shown as solid circle symbols in Figure $4 \mathrm{~b}$.

It is important to note that the Fermi level calculated from the Hall Effect electron concentration using Eq. 1 is measured from the bottom of the conduction band.

However, because the absorption edge is measured with respect to the valence band, the shift in the conduction band edge and the curvature of the valence band are included in order to compare the Fermi levels extracted from the Hall effect to those from the absorption data. In an n-type semiconductor, the conduction band shifts because of the electron-electron and electron-ion interaction. Therefore the corrected absorption edge energy represented by blue line in Figure 4a can be expressed as:

$$
E_{\mathrm{AE}}(\mathrm{E}, \mathrm{n})=E_{\text {Gap }}+E_{\mathrm{F}-\mathrm{Hall}}(\mathrm{n})-E_{\mathrm{Ie}-\mathrm{e}}(\mathrm{n})-E_{\mathrm{Ie-i}}(\mathrm{n})+\frac{\left(\hbar \mathrm{k}(\mathrm{n})^{2}\right)}{2 \mathrm{~m}_{\text {hole }}}
$$

where $\mathrm{E}_{\mathrm{Gap}}$ is the direct band gap of 2.16, $\mathrm{E}_{\mathrm{F}-\mathrm{Hall}}$ is the Fermi level calculated from Hall effect measurements, and $E_{\text {Ie-e }}(n)$ is the electron-electron (e-e) interaction calculated according to 2526 :

$$
E_{I e-e}=-\frac{2 e^{2} k_{F}}{\pi \varepsilon_{o}}-\frac{e^{2} \lambda}{2 \varepsilon_{o}}\left[1-\frac{4}{\pi} \tan ^{-1}\left(\frac{k_{F}}{\lambda}\right)\right]
$$


where $\mathrm{k}_{\mathrm{F}}=\left(3 \pi^{2} \mathrm{n}\right)^{1 / 3}$ is the Fermi wave vector, $\mathrm{n}$ is the carrier concentration, $\lambda$ is the Thomas Fermi screening parameter and is given by the following equation

$$
\lambda=2 x\left(\frac{k_{F}}{\pi a_{B}}\right)^{1 / 2}
$$

where $a_{B}$ is the Bohr radius. $E_{I e-i}(n)$ is the electron-ion (e-i) interaction calculated according to 2526

$$
E_{\text {Ie-i }}=-\frac{4 \pi n e^{2}}{\varepsilon_{o} a_{o} \lambda^{3}}=-\frac{e \hbar}{2}\left[\frac{\pi^{3} n}{\varepsilon_{o} m^{*}}\right]^{1 / 2}
$$

The last term in Eq. 4 accounts for an assumed parabolic dispersion relation of the valence band. The contributions of different terms in Eq. 4 are schematically shown in Figure 4a. As shown by the dashed curve, both the e-e and e-i interactions shift the conduction band down. The Hall electron concentration defines the position of the Fermi energy $\mathrm{E}_{\mathrm{F}}$ relative to the shifted conduction band edge. On the other hand, for a given electron concentration the optical absorption edge energy is reduced by e-e and e-i interactions. The sum of $\mathrm{I}_{\mathrm{e}-\mathrm{i}}(\mathrm{n})$ and $\mathrm{I}_{\mathrm{e}-\mathrm{e}}(\mathrm{n})$ terms ranges from $0.1 \mathrm{eV}$ in the low concentration to $0.3 \mathrm{eV}$ in the highest concentration samples. The dashed lines in Figure $4 \mathrm{~b}$ represent the electron concentration dependence of the absorption edge calculated using Eq. 4 with several values of the hole effective masses. It is seen that the absorption edge energy calculated for the hole effective mass larger than $2 \mathrm{~m}_{0}$ agrees very well with experiment.

As seen in Figure $4 \mathrm{~b}$, the shift of the absorption edge energy saturates at approximately $3.0 \mathrm{eV}$, corresponding to the electron concentration of about $5 \times 10^{20} \mathrm{~cm}^{-3}$. The saturation energy represents the position of the Fermi level stabilization energy $\left(\mathrm{E}_{\mathrm{FS}}\right)$ 
at this high electron concentration. In order to determine $\mathrm{E}_{\mathrm{FS}}$ in intrinsic $\mathrm{CdO}$, we note that both the e-e and e-i interactions shift the conduction band downward whereas $\mathrm{E}_{\mathrm{FS}}$ is affected only by the electrostatic potential of the e-i interaction. Consequently, as is shown in Figure 4a, the extrapolation to a negligible electron concentration locates $\mathrm{E}_{\mathrm{FS}}$ at $3.16 \mathrm{eV}$ above the valence band edge or at $1.0 \mathrm{eV}$ above the conduction band edge. This location of $\mathrm{E}_{\mathrm{FS}}$ is in good agreement with the pinning of the Fermi energy on the $\mathrm{CdO}$ surface as determined in a recent angle-resolved photoelectron spectroscopy (ARPES) experiment ${ }^{7}$. From the known location of the $\mathrm{E}_{\mathrm{FS}}$ at $4.9 \mathrm{eV}$ below the vacuum level one obtains the value of $5.94 \mathrm{eV}$ for the electron affinity of $\mathrm{CdO}$. This large electron affinity explains the extreme propensity of $\mathrm{CdO}$ for $\mathrm{n}$-type conductivity and points at great similarity between properties of $\mathrm{CdO}$ and previously extensively studied $\mathrm{InN}^{9}$.

\section{CONCLUSIONS}

In conclusion, we introduced point defects into high quality $\mathrm{CdO}$ thin films deposited by pulsed laser deposition by ion irradiation. We found that the carrier concentration increases as irradiation fluence increases and finally saturates at $5 \times 10^{20}$ $\mathrm{cm}^{-3}$. At the same time, we also observe that the optical absorption edge increases from $2.4 \mathrm{eV}$ for the as grown film to $3.0 \mathrm{eV}$ above the valence band in the most heavily irradiated samples. The increase in the optical absorption edge energy is attributed to the Burstein-Moss shift. We show that the saturation of the electron concentration and the absorption edge energy can be accounted for by stabilization of the Fermi energy at $\mathrm{E}_{\mathrm{FS}}$ located at $\sim 1.0 \mathrm{eV}$ above the conduction band minimum. The discrepancy between $\mathrm{He}^{+}$ 
and heavy ion irradiation in $\mathrm{CdO}$ can be explained by the large damage production rate difference between different ions and the strong ionic character of $\mathrm{CdO}$.

\section{Acknowledgments}

This work was supported by the Director, Office of Science, Office of Basic Energy

Sciences, Materials Sciences and Engineering Division, of the U.S. Department of

Energy under Contract No. DE-AC02-05CH11231. The authors would also like to thank

Willam Hansen, Jeffery Beeman, Peter Stone and Dr. Zhixun Ma for their invaluable help with target materials, film irradiation and pulsed laser deposition.

\section{References}

1 D. Ma, Z. Ye, L. Wang et al., Materials Letters 58, 128 (2003).

2 P. H. Jefferson, S. A. Hatfield, T. D. Veal et al., Applied Physics Letters 92, 022101 (2008).

3 G. Phatak and R. Lal, Thin Solid Films 245, 17 (1993).

$4 \quad$ T. K. Subramanyam, B. S. Naidu, and S. Uthanna, Applied Surface Science 169170, 529 (2001).

5 X. Li, Y. Yan, A. Mason et al., Electrochemical and Solid-State Letters 4 (9), C66 (2001).

6 J. C. Boettger, International Journal of Quantum Chemistry 107, 2988 (2007).

7 L.F.J. Piper, L. Colakerol, P.D.C. King et al., Physical Review B 78, 165127 (2008).

$8 \quad$ W. Walukiewicz, Physical Review B 37 (9), 4760 (1988).

9 S. X. Li, K. M. Yu, J. Wu et al., Physical Review B 71 (161201) (2005).

10 W. Walukiewicz, Applied Physics Letters 54 (21), 2049 (1989).

11 L. F. J. Piper, T. D. Veal, C. F. McConville et al., Applied Physics Letters 88, 252109 (2006).

12 S. X. Li, K. M. Yu, J. Wu et al., Physical Review B 376, 432 (2006).

13 P. D. C. King, T. D. Veal, P. H. Jefferson et al., Physical Review B 79, 035203 (2009).

14 S. R. Messenger, E. A. Burke, G. P. Summers et al., IEEE Transactions of Nuclear Science 46 (6), 1595 (1999).

15 S. R. Messenger, E. A. Burke, G. P. Summers et al., Progess in Photovoltaics: Research and Applications 9, 103 (2001).

16 J. F. Ziegler, J. P. Biersack, and U. Littmark, Pergamon Vol 1 (1985).

17 E. O. Kane, Journal of Physics and Chemistry of Solids 1, 249 (1957).

18 H. M. Naguib and R. Kelly, Radiation Effects and Defects in Solids 25 (1) (1975). 
H. Matzke, Radiation Effects and Defects in Solids 64 (3) (1982).

S. O. Kucheyev, J. S. Williams, J. Zou et al., Journal of Applied Physics 95 (6), 3048 (2004).

S. O. Kucheyev, J. S. Williams, C. Jagadish et al., Physical Review B 67, 094115 (2003).

R. Devanathan and W.J. Weber, Journal of Materials Research 23 (3), 593 (20089).

I. Hamberg and C. G. Granqvist, Physical Review B 30 (6), 3240 (1984).

R. E. Jones, R. Broesler, K. M. Yu et al., Journal of Applied Physics 104 (2008).

K. F. Berggren and B. E. Sernelius, Physical Review B 24 (4) (1981).

W. Walukiewicz, Physical Review B 41 (14) (1990). 


\section{FIGURE CAPTIONS}

Figure 1. (Color online) X-ray diffraction of as grown and Ne irradiated $\mathrm{CdO}$ on sapphire.

Figure 2. (Color online) Electron concentrations in $\mathrm{CdO}$ as a function of displacement damage dose using several different ions.

Figure 3. (Color online) Optical absorption edge of Ne irradiated CdO.

Figure 4. (Color online) a) Schematic defining the measured optical absorption edge, original band gap and effects of electronic interactions. b) Measured optical absorption edge compared with $\mathrm{E}_{\mathrm{F}}$ values determined from Hall effect measurements and calculated absorption edges including electron-electron interaction and assuming different electron effective masses. The Fermi level stabilization energy of about at $3.16 \mathrm{eV}$ above the valence band is obtained for both sets of measurements. 


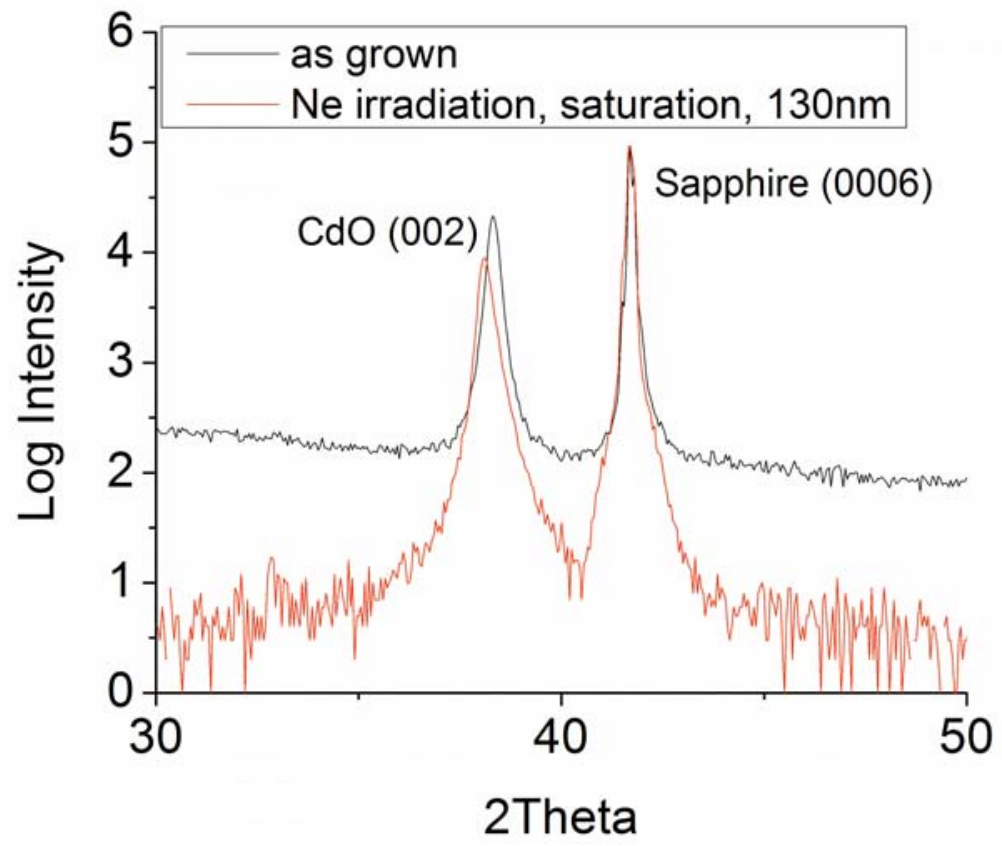

Figure 1 


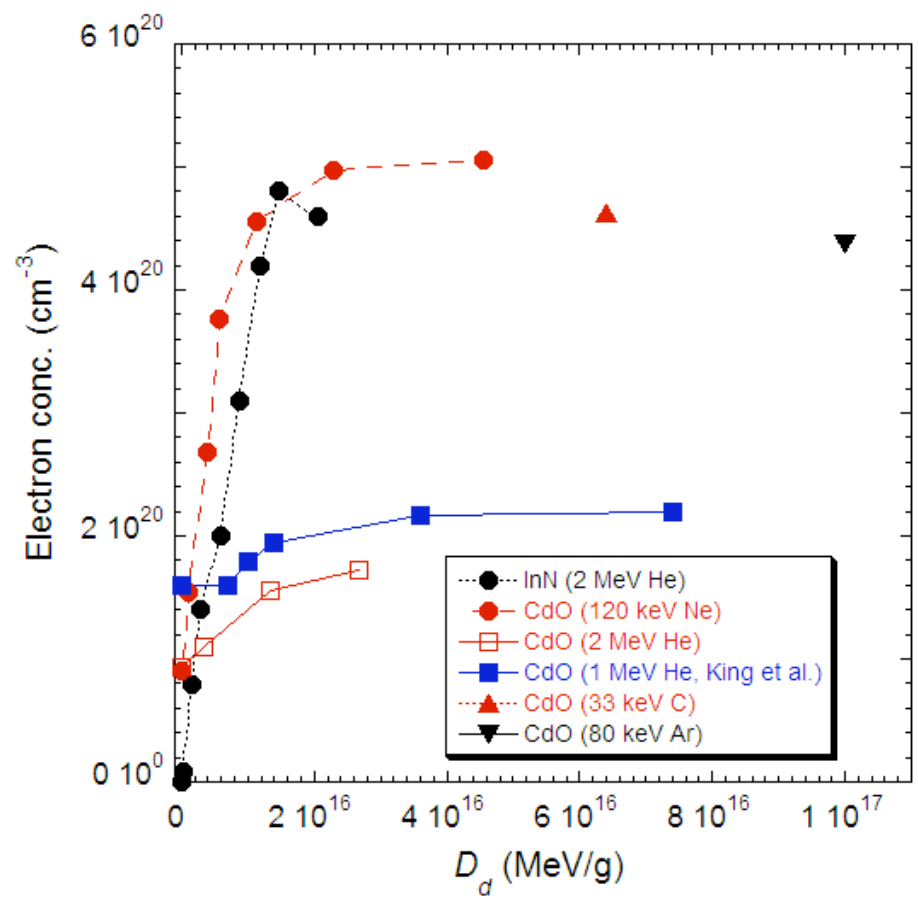

Figure 2 


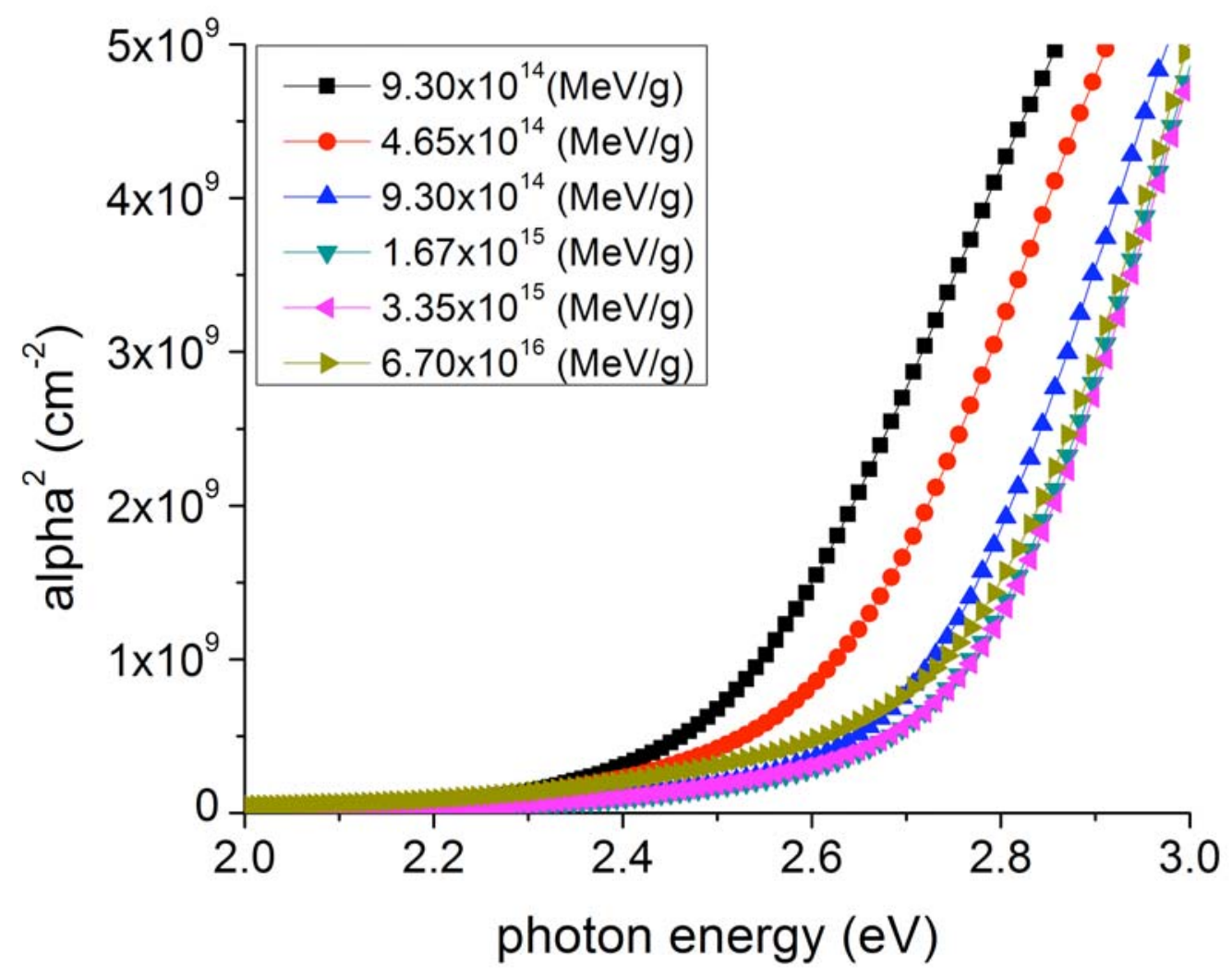

Figure 3 

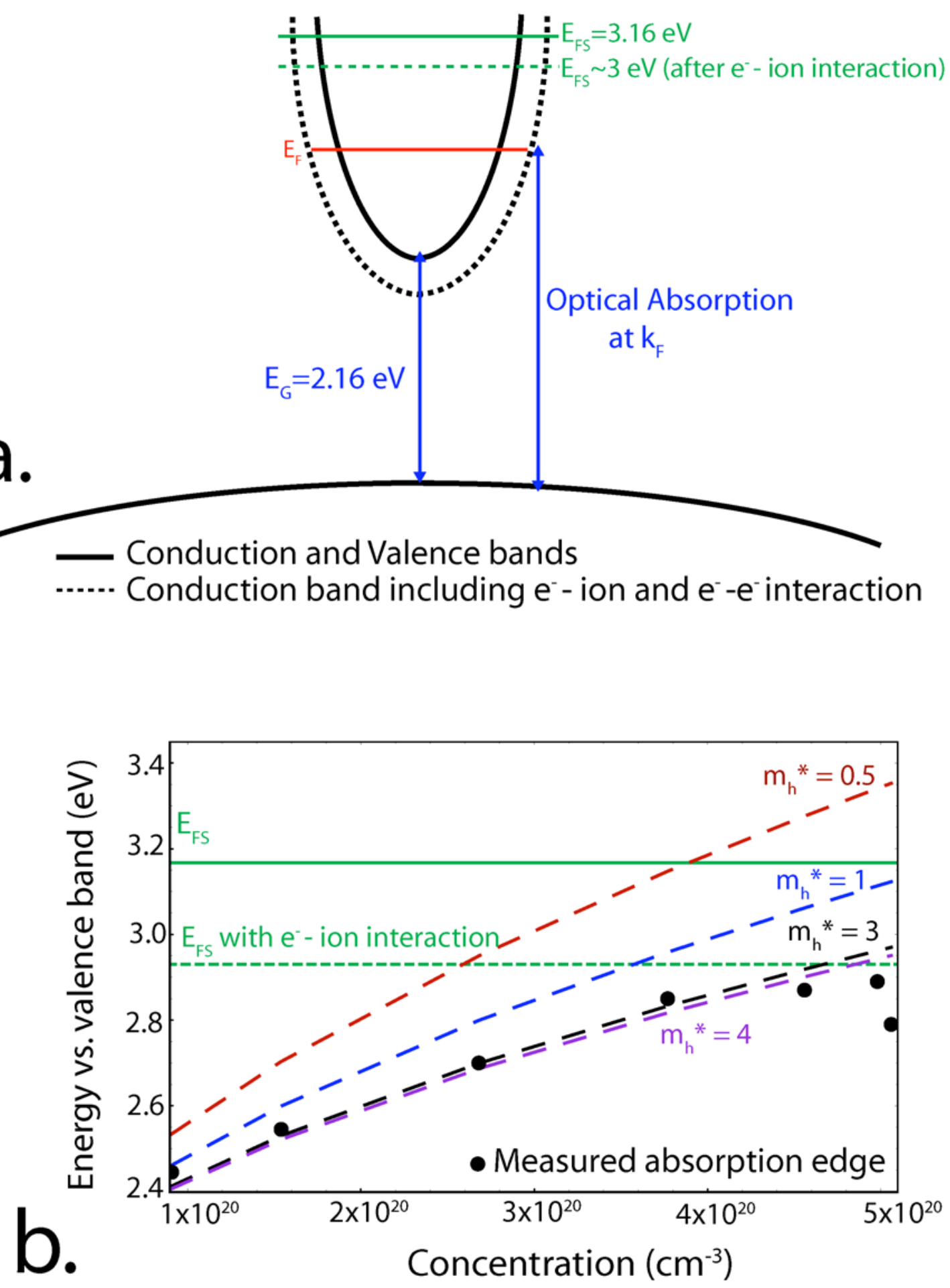

Figure 4 